IRA-International Journal of Technology \& Engineering

ISSN 2455-4480; Vol.10, Issue 03 (March, 2018)

Pg. no. 23-40.

Institute of Research Advances

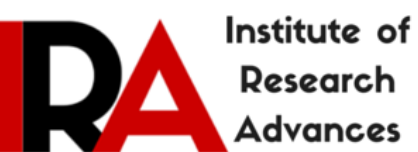

https://research-advances.org/index.php/IRAJTE

\title{
Design and Development of Plastic Filament Extruder for 3D Printing
}

\author{
Mamta H. Wankhade ${ }^{1}$, Satish G. Bahaley ${ }^{2}$ \\ ${ }^{1}$ PG Student, Mechanical Engineering Department, PRMIT\&R, Badnera, Amravati, India. \\ ${ }^{2}$ Professor, Mechanical Engineering Department, PRMIT\&R, Badnera, Amravati, India.
}

Type of Review: Peer Reviewed.

DOI: http://dx.doi.org/10.21013/jte.v10.n3.p1

\section{How to cite this paper:}

Wankhade, M.H., Bahaley, S.G. (2018). Design and Development of Plastic Filament Extruder for 3D Printing. IRA International Journal of Technology \& Engineering (ISSN 2455-4480), 10(3), 23-40. doi:http://dx.doi.org/10.21013/jte.v10.n3.p1

(c) Institute of Research Advances.

\section{(cc) BY-NC}

This work is licensed under a Creative Commons Attribution-Non Commercial 4.0 International License subject to proper citation to the publication source of the work.

Disclaimer: The scholarly papers as reviewed and published by the Institute of Research Advances (IRA) are the views and opinions of their respective authors and are not the views or opinions of the IRA. The IRA disclaims of any harm or loss caused due to the published content to any party.

Institute of Research Advances is an institutional publisher member of Publishers Inter Linking Association Inc. (PILA-CrossRef), USA. The institute is an institutional signatory to the Budapest Open Access Initiative, Hungary advocating the open access of scientific and scholarly knowledge. The Institute is a registered content provider under Open Access Initiative Protocol for Metadata Harvesting (OAI-PMH).

The journal is indexed E $^{\circ}$ included in WorldCat Discovery Service (USA), CrossRef Metadata Search (USA), WorldCat (USA), OCLC (USA), Open J-Gate (India), EZB (Germany) Scilit (Switzerland), Airiti (China), Bielefeld Academic Search Engine (BASE) of Bielefeld University, Germany, PKP Index of Simon Fraser University, Canada. 


\begin{abstract}
$3 D$ printing is a form of additive manufacturing technology where a three dimensional object is created by laying down successive layers of material. It is mechanized method whereby $3 D$ objects are quickly made on a reasonably sized machine connected to a computer containing blueprints for the object. As $3 D$ printing is growing fast and giving a boost to product development, the factories doing $3 D$ printing need to continuously meet the printing requirements and maintain an adequate amount of inventory of the filament. As the manufactures have to buy these filaments from various vendors, the cost of $3 D$ printing increases. To overcome the problem faced by the manufacturers, small workshop owners, the need of $3 D$ filament making machine arises. This project focuses on designing and fabricating a portable fused deposition $3 D$ printer filament making machine with cheap and easily available components to draw $1.75 \mathrm{~mm}$ diameter $A B S$ filament
\end{abstract}

Keywords: ABS, 3D printing, Extrusion, Single Screw Extruder

\title{
Introduction
}

Due to the large scope of 3D printing this technology has experienced in the recent decades a great development. The access to 3D printers is becoming easier as the prizes are going down. Nowadays both companies and regular users can develop their own parts in a relatively simple and quickly way. That is why there is more and more interest in evolving this technology which has already revolutionized manufacturing processes.

Today there are plenty options when choosing a printer and a lot of different companies that manufacture and sell these printers. But most of the 3D printer is based on the fused Deposition Method, which uses mostly PLA and ABS as the printer material. This extrusion based 3D printer uses a wired filament of diameter $1.75 \mathrm{~mm}$ or $3 \mathrm{~mm}$ for printing. So most of the manufacturing units, companies, colleges who have the 3D printer in-house are dependent on 3rd party supplier, and need to buy the $3 \mathrm{D}$ printer filament from these suppliers.

This paper will make you go through the design and development of a portable 3D printer filament making machine for small manufacturing units and colleges.

The new designed portable filament making machine should be able to make the wired filament from the plastic granules.

With the development of the portable filament making machine, the dependency of the manufacturing units and other organization on 3rd party supplier for filament will be reduced.

\section{Objectives}

1. To design and develop a plastic filament extruder for 3D printing

2. The focus was specifically on creating $1.75 \mathrm{~mm}$ diameter filament from ABS pellets.

3. To develop a 3D printing filament making extruder that can be used by small scale manufacturing units, companies, colleges who have portable 3D printer in-house.

4. To Perform design calculations to base the development of filament making extruder

\section{Motivation}

$3 \mathrm{D}$ printing is growing technology and is used worldwide. 3D printing requires filament to process and the cost of filament governs the cost of 3D printing product. Filament extrusion machines are usually available for industrial use, capable of creating hundreds of feet of long filament in a day. So these filaments are expensive for many end users.

This work will make easily available filament extruder to small scale industries and colleges. 


\section{Option of Design}

\section{Horizontal 3D filament making machine}

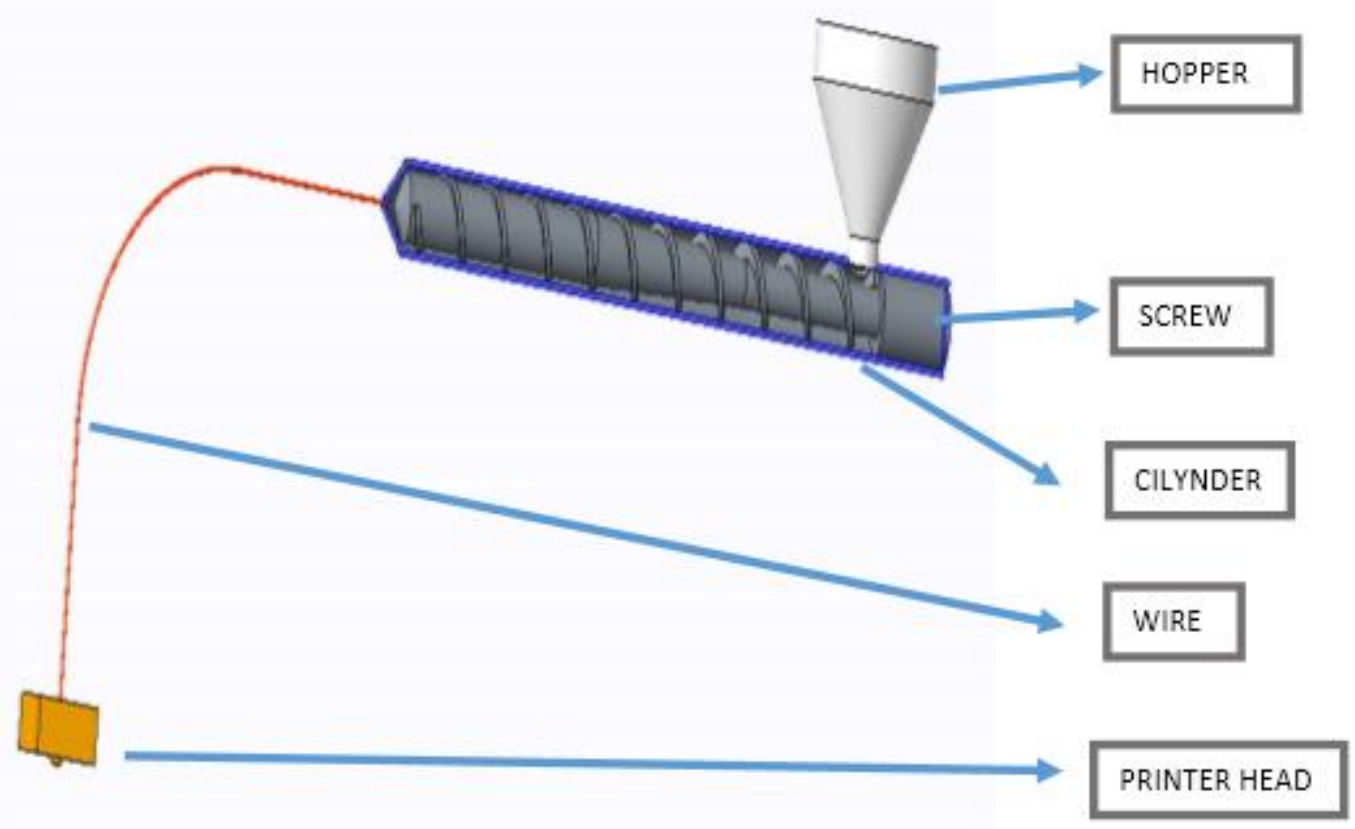

Figure 1 Horizontal 3D filament making machine

Most 3D printers use a wire as feed material, so the first option is to develop an extruder that permits the manufacture of the wire. This allows us to start the process of printing from plastic granulate.

The process starts feeding the hopper with the plastic. The screw drags and breaks granulate. The extrusion process continues until the material comes out of the nozzle creating he wire which will be guide by a plastic tube into the printer's head.

Apparently this option is the simplest one but there are some problems to be solved. The biggest problem is when the extruder stops working all the plastic inside the canal lowers its temperature and solidifies. So is not possible to start the process again. Furthermore, as the wire comes out with a high temperature and in a melted way, the material gets stuck into the tubes walls and the printing process is not uniform and it can block the tube.

\section{Advantage}

Easy design

\section{Problems/disadvantages:}

When the process is stopped, the plastic in the tube that connects the extruder solidifies. This makes impossible to start another impression. As the plastic comes out at a very high temperature of the extruder, it could stay stuck in the tube walls

\section{Equipment Description}

\section{Screw}

There are two basic characteristics that the screw should satisfy in order to perform his function correctly. It has to be hard enough to bear with the possible erosion and to be able to handle with high temperatures. 
The high temperatures will be caused by the movement that the screw has, the friction against the cylinder and the heating system.

The material chosen for the screw is steel F-174, which is a nitriding steel. This material is typically used in extruders screws and cylinders and reaches a vickers hardness of 1048-1064 HV. In addition, it is able to handle with the high temperatures reached inside the extruder, which will be around $200^{\circ} \mathrm{C}$. Steel pieces which have been treated with a nitriding process are usually prepared to stand temperatures up to $500^{\circ} \mathrm{C}$. Also the nitriding process gives the piece an extra layer of protection against the corrosion.

For the properly development of the extrusion process it is necessary that the screw surface is as smooth as possible, to avoid friction and to allow the plastic slide on it.



Figure 2 Screw Geometry details

The diameter we decide to work with is $25 \mathrm{~mm}$ and for the relation between the length and the diameter we choose, 12/1. The reasons to take these measures and not any others are based basically on the fact that the screw has to be the smallest size possible without increasing very much the price. So we consider $25 \mathrm{~mm}$ to be the smallest diameter with reasonable price and with precise usefulness. In addition, we choose 12/1 for the relation L/D because we consider $300 \mathrm{~mm}$ the maximum length keeping a light screw in terms of weight, taking into account that if the relation L/D is bigger, the price will be lower.

Therefore, I have defined the first parameters of our screw $\mathrm{D}=25$ and $\mathrm{L}=300$. Also they have been taken into account other options of diameters and length.

\section{Number of channels}

The first step to be taken in the process of design a screw is deciding the number of channels on it that is, deciding the number of threads. In applications where a large flow is required, screws can be used with two or more threads, but in our case the flow is very small so we will use a screw with a single thread. So the number of channels for our extruder $\mathrm{m}=1$. 




Figure 3 Number of channels of Screw

\section{Helix Angle}

Also one of the first things we can determine of our extruder is the helix angle of the screw. For general purpose screws, the gap between 2 crests or the pitch ( $t$ ) usually coincides with the diameter. So

$$
\mathrm{t}=\mathrm{D}=25 \mathrm{~mm}
$$

So the helix angle is

$$
\begin{gathered}
\varphi=\tan -1 \frac{\mathrm{t}}{\pi \mathrm{D}} \\
\varphi=\tan -1 \frac{25}{\pi * 25} \\
\varphi=17.65^{\circ}
\end{gathered}
$$

\section{Ridge width}

The width of the ridge is defined by the diameter of the screw as it exists a relation between them, $\mathrm{e}=0.12 * \mathrm{D}$

So e $=.12 * 25$

$\mathrm{e}=3 \mathrm{~mm}$

\section{Screw lengths}

For amorphous thermoplastic, the feeding zone is between $20 \%$ and $25 \%$ of the screw length, the compression zone between $32 \%$ and $38 \%$ and for the metering zone between $40 \%$ and $45 \%$.

We based our decision of the zones lengths on the percentage from the total length that normally has each zone.

The percentages used in each zone are obtained as follows:

$$
\begin{aligned}
& \%=\frac{\%}{\% 1+\% 2+\% 3} \\
& \text { L1 \%= } \frac{20}{20+32+40}=0.217 \\
& \text { L2 } \%=\frac{32}{20+32+40}=0.348 \\
& \text { L3\% }=\frac{40}{20+32+40}=0.435
\end{aligned}
$$

Feeding Zone Length: $\mathrm{L} 1=0.217 * 300=65 \mathrm{~mm}$

Compression Zone Length: L2 $=0.348 * 300=105 \mathrm{~mm}$

Metering Zone Length: $\mathrm{L} 3=0.435 * 300=130 \mathrm{~mm}$

\section{Channel depth and screw clearances}

The clearances inside the screw and with the cylinder are also defined by the diameter I have chosen. The channel depth $\mathrm{h} 1$, is the space between the cylinder and the soul of the screw. It is related with the screw diameter with the equation

$$
\begin{aligned}
& \mathrm{h} 1=0.2 * \mathrm{D} \\
& \mathrm{h} 1=5 \mathrm{~mm}
\end{aligned}
$$


The filet clearance is the space between the thread and the interior surface of the cylinder. It should be small enough to avoid the plastic to come back while extruding.

The equation to calculate it is:

$$
\begin{aligned}
& \delta=0.002 * \mathrm{D} \\
& \delta=0.05 \mathrm{~mm}
\end{aligned}
$$

The depth of the channel at the end of the screw is defined by the compression ratio (Z). The compression ratio relates the depth of the channel at the beginning and at the end of the screw.

\section{Barrel or Cylinder}

Just as for the screw, the material chosen for the cylinder is steel F-174, for the same reasons. The cylinder must also be able to handle with high temperatures and be hard enough to resist degradation due to the friction generated between the inner face of the cylinder and the plastic flow.

The cylinder is the part in charge of keeping the material inside while going throughout the screw. For this reason, its inner diameter is the sum of the screw diameter and the clearance calculated above, to a total of $25.05 \mathrm{~mm}$.

Considering tolerance and according to the availability of standard tube, 1" ID tube meets our requirement, so we have selected 1"ID tube for the cylinder.

\section{Barrel Extension}

Barrel Extension material is same as Barrel for the same reasons. It is manufactured by turning operation. It is welded to the end of the Barrel. This extension is used to couple barrel piece to die and to give sufficient thickness to fit the secondary heater.

In addition, $4 \mathrm{~mm}$ thick aluminum strainer is attached to smoothen the flow and $3 / 8$ " nut to control the flow.

\section{Die or Nozzle}

The material most commonly used for die is brass because it has to withstand high temperatures. Likewise, is a good conductor of heat, quality that is needed to heat fast and uniform the nozzle as the printing material needs to be printed around $200^{\circ} \mathrm{C}$. Brass is one of the material with best characteristics and this is why we are choosing it for the nozzle. The nozzle is also one of the most important elements of extruder, as it defines the final shape of the plastic. Between its characteristics we are going to remark its hardness and the fact that it perfectly keeps its conditions for a long period of time. Also, it doesn't get affected by the external conditions. Its characteristics make it one of the best materials in the market but with a lower price. The die that is used is M12 Brass plug with $2 \mathrm{~mm}$ hole.

\section{Hopper}

Hopper is made up of stainless steel sheet metal.

There are no specifications for hopper design. Its size varies depending on the application or quantity of production. So the hopper design is just to fulfill the requirement of this project.

Hopper is designed as gravity fed hopper. Hopper is wedge type and the flow of solid in the hopper is mass flow. It is cut and manufactured from 6" 4 "

\section{Motor}

The motor for the system is a $55 \mathrm{RPM}$ motor with stall torque of $53 \mathrm{lb}$-in $(61 \mathrm{~kg}-\mathrm{cm})$. This motor is controlled by a PWM (Pulse Width Modulation) speed controller. This controller is wired in series with the 
power source from $24 \mathrm{~V}$ supply and the motor. This was the simplest control system. It is a variable speed control system with the RPM is selected by varying the duty cycle

\section{Heater system design}

Heaters are located along the barrel, with thermocouples in each zone to control the heaters and barrel temperature. The heaters cover as much barrel surface area as practical, minimizing hot and cold spots along the barrel length. In an individual extruder temperature zone, there may be one, two, or three heater bands with one thermocouple controlling them. Assume the heater band closest to the thermocouple burns out; the other two heater bands have to supply all the external energy required, creating the possibility that the area is hotter near the two heater bands that are working. In the event, the band farthest from the thermocouple burns out, the barrel area under the burnt-out heater is anticipated to be cooler than areas where the heaters are functioning properly near the controlling thermocouple. Burnt-out heater bands should be replaced as soon as possible to assure uniform heat input.

\section{Temperature Zone Control}

Each extruder temperature zone has at least one heater and possibly multiple heaters controlled by a thermocouple. A signal from the thermocouple communicates with the controller, indicating whether the heater is to be turned on or off. For the controller and heaters to function properly, the thermocouple must operate properly. A faulty thermocouple with an open circuit indicates the temperature is low, resulting in the heaters staying on and causing substantial overheating. A closed thermocouple indicates the temperature is high; heaters remain off and the temperature zone cools. If a thermocouple is not responding properly, it must be replaced.

The thermocouple well in the barrel should be at least 1.2 inches $(30 \mathrm{~mm})$ deep and installed away from the heaters. Never sandwich the thermocouple between the heater and the barrel wall; the thermocouple will be responsive to the heater temperature and not the barrel temperature.

We have used 35 x $30 \mathrm{~mm}, 150$ Watt heating band.

\section{Fixing system}

What we have called fixing system; it is nothing but the pieces we have designed to set our extruder. This fixation system is responsible for holding the extruder and the rest of the pieces. In addition, the fixing system will hold the fans that cool the filament coming out of die.. Therefore, the pieces we have to design should perform simultaneously three different functions. To start designing the pieces we look at the extruder which we are going to assemble. In addition, the heater system is designed in a way so that the fixing system has to be allocated between both of the heater pieces. So we have designed the fixing system that will cover all the extruder parts. We have used Mild steel square-shaped tube for fixing system designed.

\section{EXTRUDER PRODUCTION CALCULATIONS}

\section{Volumetric Flow}

Production expressed as volumetric flow, Q, is the result of three different types of flow.

A) Drag flow is the largest component caused by turning the screw.

B) Pressure flow, is the component that opposes the flow system. Flow filtration is produced by the loss of material between the clearances of the screw cylinder.

The volumetric flow $Q$ can be calculated with this equation:

Where:

$$
\mathrm{Q}=\left(\frac{\alpha K}{K+\gamma+\beta}\right) \eta
$$

Drag flow coefficient: ${ }^{\alpha}$

Pressure flow coefficient: ${ }^{\beta}$ 
Filtration flow coefficient: ${ }^{Y}$

Head geometrical constant: $\mathrm{K}$

Spindle speed: $\eta$

\section{Drag Flow Coefficient}

The Drag flow is the component caused by the turning screw. The plastic enclosed between the spindle and the cylinder is forced to advance in axial direction. It can be calculated with this equation:

$$
\alpha=\frac{\pi * m * D * h *\left(\frac{t}{m}-e\right) * \cos ^{2} \phi}{2}
$$

Where:

Screw diameter: $\mathrm{D}=25 \mathrm{~mm}$.

Pitch: $\mathrm{t}=25 \mathrm{~mm}$.

Initial channel depth: $\mathrm{h} 1=5 \mathrm{~mm}$.

Helix angle: $\phi=17.65$.

Ridge width: $\mathrm{e}=3$

Number of channels $m=1$.

Thereupon the drag flow we will have:

$$
\alpha=3922.57 \mathrm{~mm} 3 \text { or } 3.92 \mathrm{~cm} 3
$$

\section{Pressure Flow Coefficient}

Pressure flows appears against the main flow. It is very important to design properly the screw so the pressure flow is lower that the drag flow, otherwise the extruder won't have any production flow.

Where:

$$
\beta=\frac{m * h^{3} *\left(\frac{t}{m}-e\right) * \sin \phi * \cos \phi}{12 * L}
$$

Screw length: $\mathrm{L}=300 \mathrm{~mm}$

The rest of the parameters are the same that the ones we used to calculate the drag flow coefficient.

$$
\beta=.2207 \mathrm{~mm} 3 \text { or } 2.209 * 10^{-4} \mathrm{~cm} 3
$$

\section{Filtration Flow Coefficient}

The filtration flow coefficient represents the portion of fluid that seeps between the crest of the screw and the cylinder wall.

Where:

$$
\gamma=\frac{\pi^{2} * D^{2} * \delta^{3} * \tan \phi}{10 * e * L}
$$

Filet clearance: $\delta=0.05 \mathrm{~mm}$

The rest of the parameters are the same as the previous ones

$$
\gamma=2.725 * 10^{-05} \mathrm{~mm} 3 \text { or } 2.7258 * 10^{-8} \mathrm{~cm} 3
$$

\subsubsection{Head Geometrical Constant}

$\mathrm{d} 0=\mathrm{D}-2 * h=12 \mathrm{~mm}$ (here $\mathrm{D}$ is nozzle outer diameter)

$$
\mathrm{d} 1=2 \mathrm{~mm}
$$

To determine the constant $\mathrm{K}$, the head is divided into successive zones of different settings, setting for each of these areas a different constant ki. The head is dividing in two different zones, one conical and the other one cylindrical.

To the conical section:

$$
\mathrm{k}_{1}=\frac{3 * \pi * \mathrm{~d} 1^{3} * \mathrm{~d} 0^{3}}{128 * L *\left(\mathrm{~d} 1^{2}+\mathrm{d} 1 * \mathrm{~d} 0+\mathrm{d} 0^{2}\right)}
$$


And

To the Cylindrical Section

$$
\mathrm{k} 2=\frac{\pi * \mathrm{~d}^{4}}{128 * \mathrm{~L}}
$$

Since the nozzle for the extruder is being made manually from a 0.5 inch threaded brass plug. So it is in a cylindrical shape, so we will only calculate the Cylindrical Section.

That is the

$\mathrm{L}$ is the length of the nozzle, which is $25 \mathrm{~mm}$ or $2.5 \mathrm{~cm}$

$$
\mathrm{k} 2=0.03195
$$

So the geometrical constant $\mathrm{K}$ is 0.03195

\section{Screw Turning Speed and Flow Rate}

Since the max rated RPM of the motor is 55 , considering a factor of safety of 2 .

Let the speed of the screw be 30 RPM

Where

$$
\begin{aligned}
& \mathrm{Q}=\left(\frac{\alpha * K}{K+\gamma+\beta}\right) \eta \\
& \eta=\frac{Q}{\frac{\alpha * K}{K+\gamma+\beta}}
\end{aligned}
$$

Flow rate: $\mathrm{Q}$

Drag flow coefficient: $\alpha=2008.35 \mathrm{~mm} 3$

Pressure flow coefficient: $\beta=5.650 * 10-3 \mathrm{~mm} 3$

Filtration flow coefficient: $\gamma=1.11 * 10-05 \mathrm{~mm} 3$

Head geometrical constant: $\mathrm{K}=0.03195$

$$
\begin{aligned}
\mathrm{Q} & =\left(\frac{3922.57 * 0.03195}{0.03195+2.725 * 10^{-5}+.2207}\right) 30 \\
\mathrm{Q} & =\frac{3759.78}{0.2526} \\
\mathrm{Q} & =14879.78 \mathrm{~mm} 3 / \mathrm{min} \\
\mathrm{Q} & =248 \mathrm{~mm} 3 / \mathrm{sec}
\end{aligned}
$$

\section{Required Power}

The power required symbolizes the speed with which a job is carried out. This is an approximation to the power we need to add to the extruder so it can melt the plastic. It can be calculated using the following energy balance:

Where

$$
\mathrm{P}=\rho * Q * C *\left(T_{m}-T_{0}\right)
$$

Density: $\mathrm{\rho}=1.07 * 10-6 \mathrm{~kg} / \mathrm{mm} 3$

Flow rate: $\mathrm{Q}=248 \mathrm{~mm} 3 / \mathrm{sec}$

Heat capacity of the material: $\mathrm{C}=1300 \mathrm{~J} / \mathrm{kg} \mathrm{K}$

Outlet temperature: $\mathrm{Tm}=200^{\circ} \mathrm{C}$

Inlet temperature: $\mathrm{T} 0=20^{\circ} \mathrm{C}$

$$
\mathrm{P}=62.09 \mathrm{~W}
$$




\section{Hopper Calculations}

\section{Stresses in Hoppers}

Density: ${ }^{P}=1070 \mathrm{~kg} / \mathrm{m} 3$

Wall friction angle varies from 15 to 30 degrees for carbon stainless steel. So we have taken 20 degree $\varphi=20$

$\varphi=$ Angle of wall friction

The coefficient of wall friction is

$\mu=\tan (20)=0.3639$

Compressive Normal Stresses

$\mathrm{pv}=\frac{\rho \mathrm{gD}}{4 \mu \mathrm{Kg} \mathrm{c}}\left(1-\exp \left(-\frac{4 \mathrm{z} \mu \mathrm{K}}{\mathrm{D}}\right)\right)$

$\mathrm{gc}=$ gravity constant conversion factor $=1 \mathrm{Kg} \mathrm{m} / \mathrm{N}$ s2

$\mathrm{K}=$ the Janssen Coefficient $=0.4$

The hopper which we have designed has a square of $80 \mathrm{~mm}$.

So the equivalent diameter will be .

$\frac{\pi}{4} * \mathrm{D} 2=\mathrm{a} 2$

$\stackrel{4}{\mathrm{D}}=91 \mathrm{~mm}=.091 \mathrm{~m}$

$\mathrm{pv}=\frac{1070 * 9.8 * 0.091}{4 * 3639 * 4 * 1}\left(1-\exp \left(-\frac{4 * .1 * 3639 * .4}{.091}\right)\right)$

$\mathrm{pv}=774.56 \mathrm{~N} / \mathrm{m} 2$

To estimate the normal stress on the wall we apply Janssen's assumption

$$
\begin{aligned}
& \mathrm{Pw}=\mathrm{KPv} \\
& \mathrm{Pw}=.4 * 774.56 \\
& \mathrm{Pw}=309.825 \mathrm{~N} / \mathrm{m} 2
\end{aligned}
$$

\section{Hopper volume calculations}

To calculate the volume, we have approached the inner cavity of the hopper dividing it into two zones, one cubical and one conical. So that the total volume will be the sum of these two areas
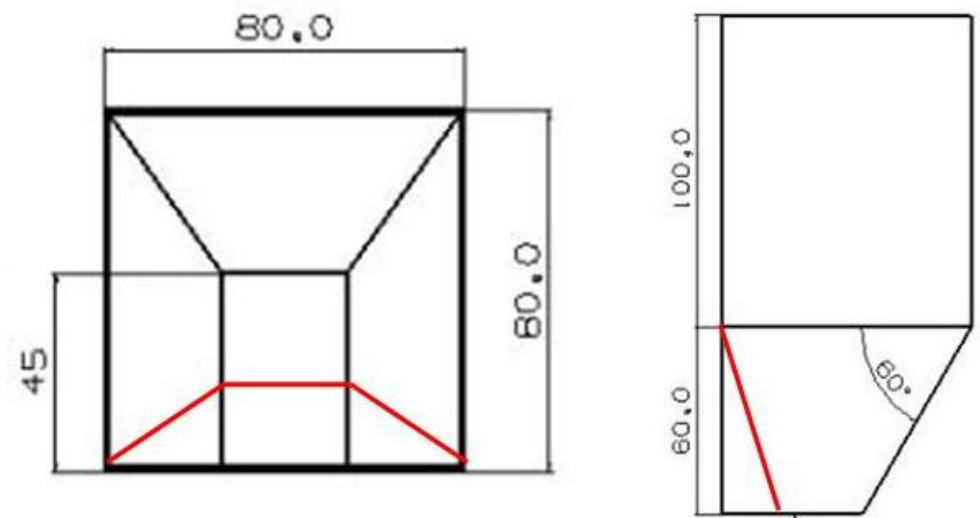

Figure 4 Hopper diagram (Red lines to show Hopper lower part division for volume calculation)

Volume of the rectangular section

$\mathrm{V}=\mathrm{L} * \mathrm{~B} * \mathrm{H}$

$\mathrm{V}=100 * 80 * 80$

$\mathrm{V}=640000 \mathrm{~mm} 3$

Volume of the trapezoid 
Volume of trapezoid $=\frac{1}{3} *(\mathrm{~A} 1+\mathrm{A} 2+\sqrt{\mathrm{A}} 1 * \mathrm{~A} 2) * \mathrm{~h}$

Volume of trapezium $=\frac{1}{3} *(900+6400+\sqrt{ } 900 * 6400) * 60$

Volume of trapezium $=\frac{1}{3} * 9700 * 60$

Volume of the trapezium $=194000 \mathrm{~mm} 3$

As the hopper is flat from one side so the volume from the front side excluding the trapezium

So the volume of triangular section is

$\mathrm{V}=(.5 * 15 * 30 * 60)$

$\mathrm{V}=13500 \mathrm{~mm} 3$

So the total volume of the hopper is

$\mathrm{V}=(640000+194000+13500) \mathrm{mm} 3$

$\mathrm{V}=847500 \mathrm{~mm} 3$

Considering that the flow rate of the extruder is $\mathrm{Q}=248 \mathrm{~mm} 3 / \mathrm{sec}$. We can calculate the time, in hours, that the extruder can be working without being refilled.

$$
\begin{aligned}
\mathrm{T} & =\frac{\mathrm{V}}{\mathrm{Q} * 3600} \\
\mathrm{~T} & =\frac{847500}{248 * 3600} \\
\mathrm{~T} & =0.95 \text { hours }
\end{aligned}
$$

So the hopper needs to be refilled after every 0.95 hours so as to the extruder works continuously.

\section{Calculation of $\mathbf{b}_{\min }$ for Free Flowing Bulk Solid}

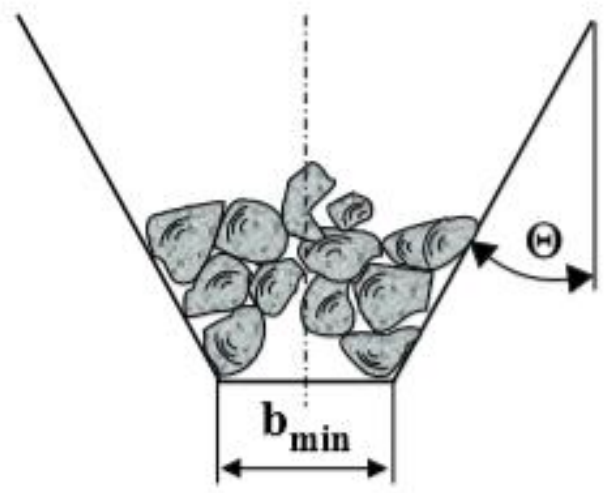

Figure 5 Hopper design for free flowing Bulk solid

The slot of the hopper is rectangular so

$\mathrm{b}_{\min }=5 \mathrm{~d} 0 \sqrt{3}$

where

$\mathrm{d} 0=$ upper particle size $=3 \mathrm{~mm}$

$\mathrm{b}_{\min }=5 * 3 * \sqrt{3}$

$\mathrm{b}_{\min }=25.98 \mathrm{~mm}$

In our design we have taken $b_{\min }=30$ for hopper to free flow of bulk solid.

\section{Heating calculations}

\section{Melting Power needed}

I have already chosen our resistances, ensuring that allow us to obtain the required temperature of $200^{\circ} \mathrm{C}$, as they can reach $250^{\circ} \mathrm{C}$. However, we think is also good to have an approximation of the power that they will need. 
$\mathrm{W}=\rho * \mathrm{~V} * \mathrm{C} * \Delta \mathrm{T}$

Density: ${ }^{P}=1.07 * 10-6 \mathrm{~kg} / \mathrm{mm} 3$

Heat capacity of the material: $\mathrm{C}=1300 \mathrm{~J} / \mathrm{kg} \mathrm{K}$

To calculate the volume enclosed inside the extruder we depreciate the volume occupied by the screw helix. In this way, the volume of plastic will be the difference between the volume of the chamber inside the cylinder and the volume occupied by the screw.

The volume of barrel cylinder is:

$\mathrm{V}=\pi * \mathrm{l} * \mathrm{r}^{2}$

Where

Length $=1=300 \mathrm{~mm}$

Radius $=12.5 \mathrm{~mm}$

$\mathrm{V}=147262.15 \mathrm{~mm} 3$

$\mathrm{W}=36871.5 \mathrm{~J}$

\section{Working and Results}

The main part of the extruder is a barrel containing a screw (also sometimes referred to as an "auger") which is connected to a heater toward its far end. On the other end, the screw is connected to an electric motor which will via mechanical action, transport the resin pellets through barrel towards the heater. Pellets are gravity fed continuously from a hopper. As the motor is continuously driving the auger, the resin pellets are pushed into the heater. The ABS pallets will get soften and melt because of the heat from heating band that is evenly distributed by using asbestos ribbon and are then pushed mechanically through a die. Pushing the soft ABS through the die will cause it to form a continuous filament strand with the diameter of die. Screw is used to control the flow and strainer is used to smoothen the flow. Air fan cool down mechanism is used to cool down the filament after coming from the die. The filament will be stretched out of die till the end wheel and it will be rolled using $12 \mathrm{~V}$ DC motor.

\section{Extruder parts CAD and Real parts images}
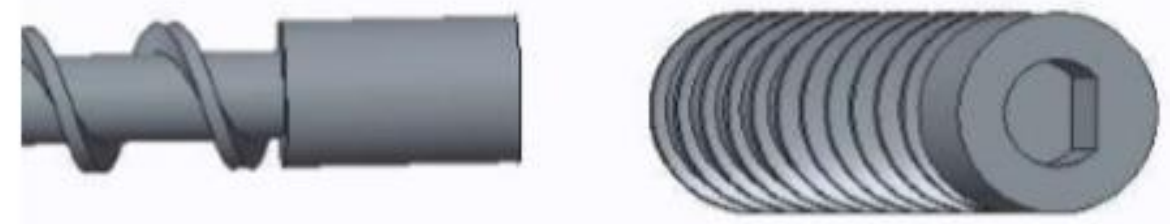

\section{Image1 Screw CAD Model}

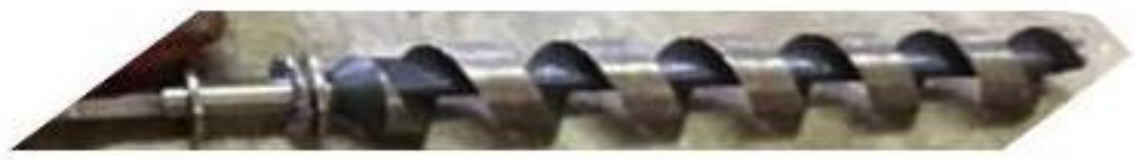

Image2 Screw 


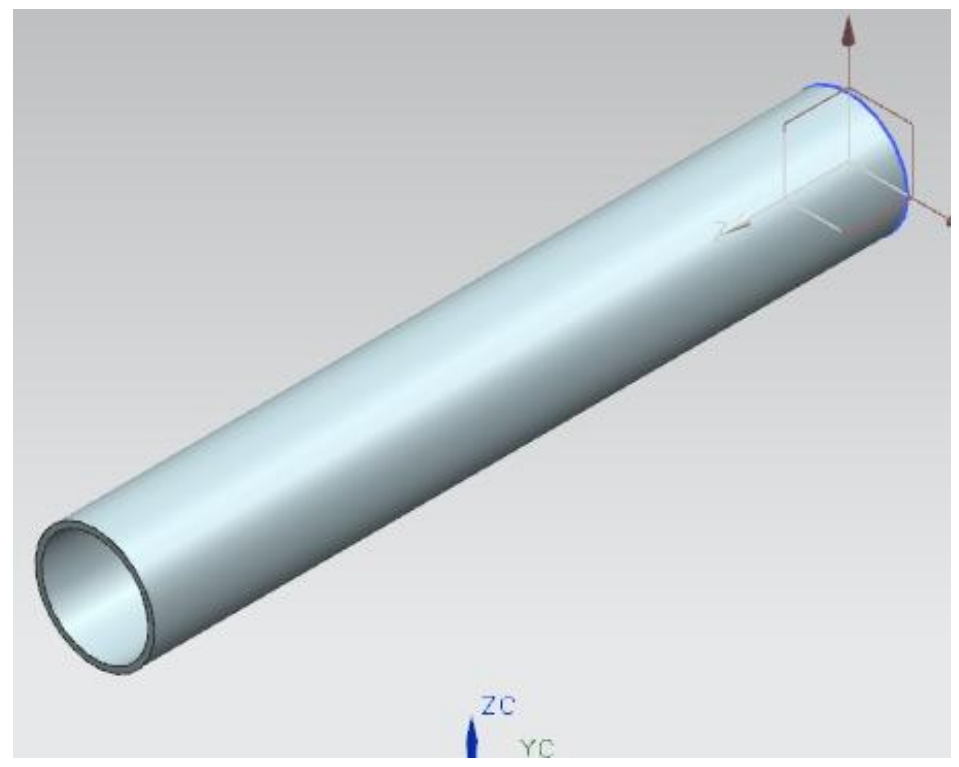

Image 3 Cylinder CAD Model

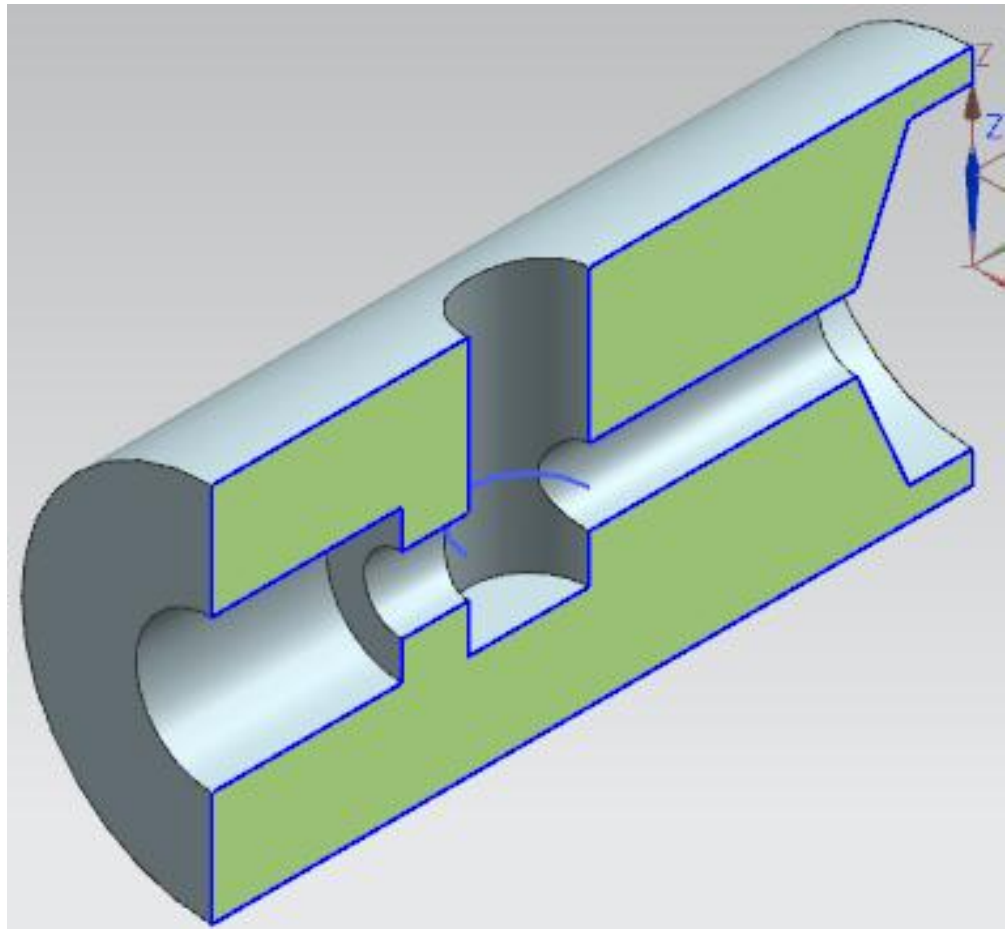

Image 4 Barrel extension CAD Model 


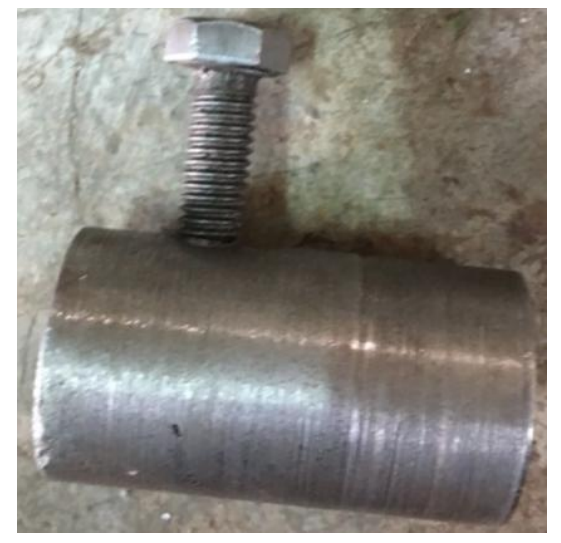

Image 5 Barrel Extension with Screw

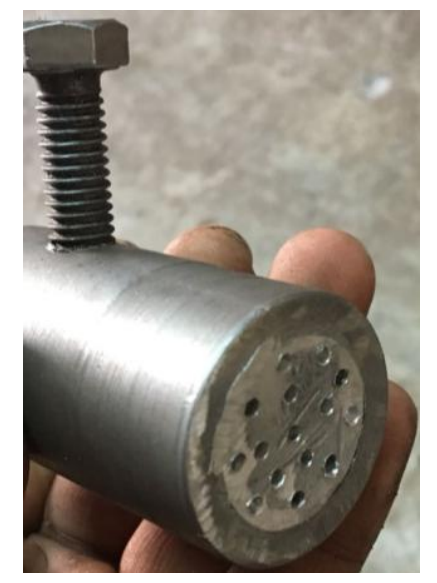

Image 5 Barrel Extension with Strainer

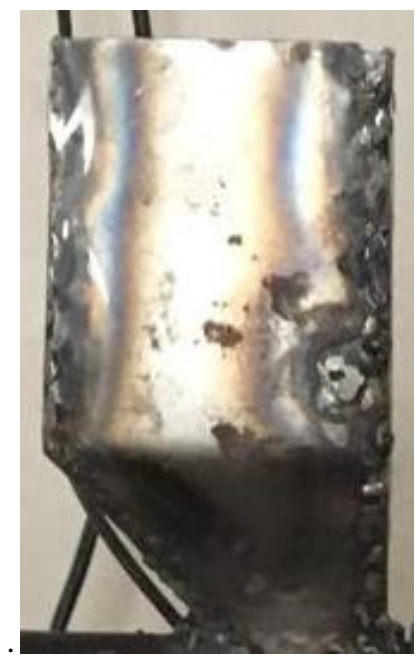

Image 7 Hopper

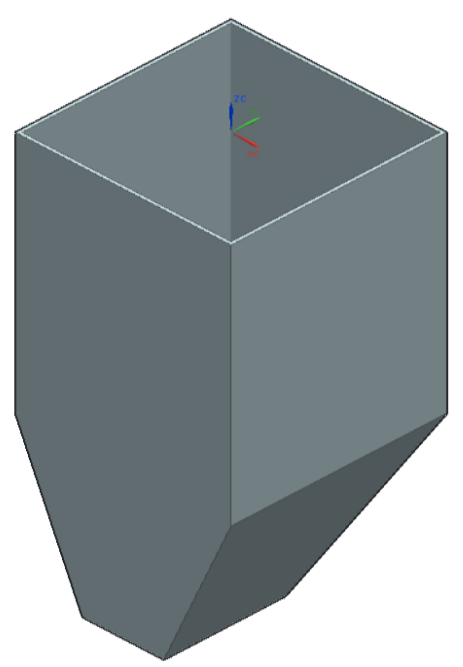

Image 8 Hopper CAD model 


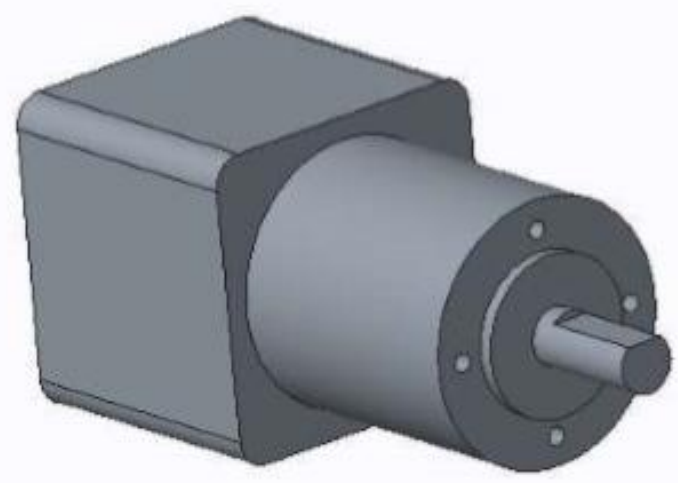

Image 9 Motor CAD Model

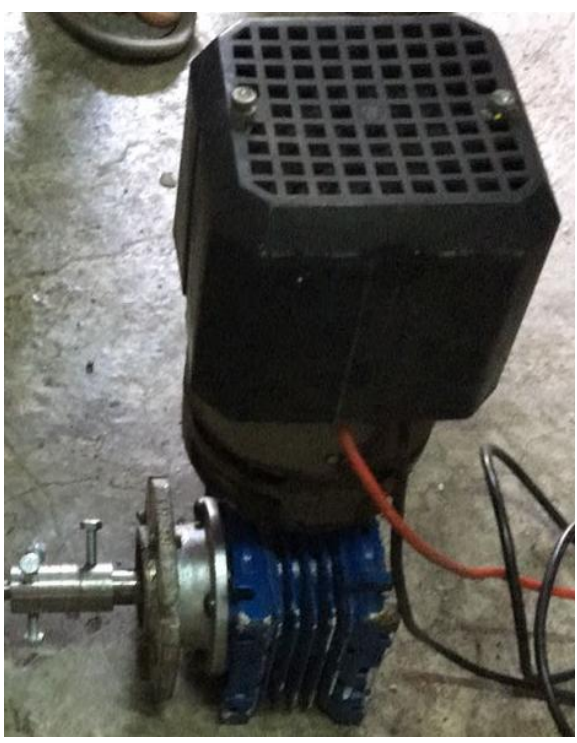

Image 10 600W Worm Geared Motor

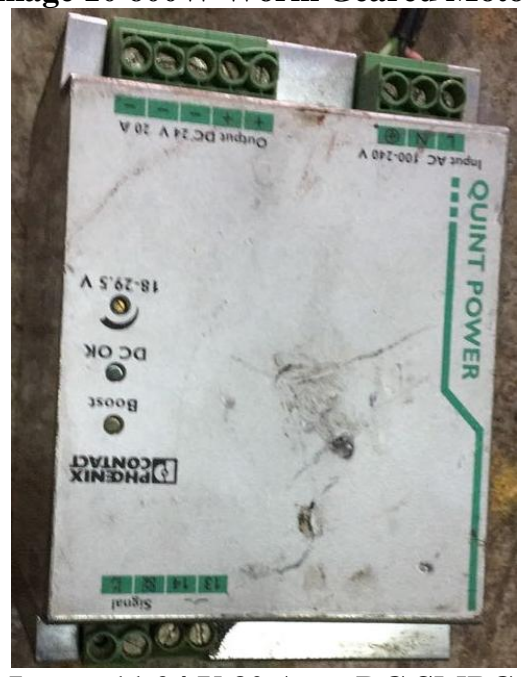

Image 1124 V 30 Amp DC SMPC 


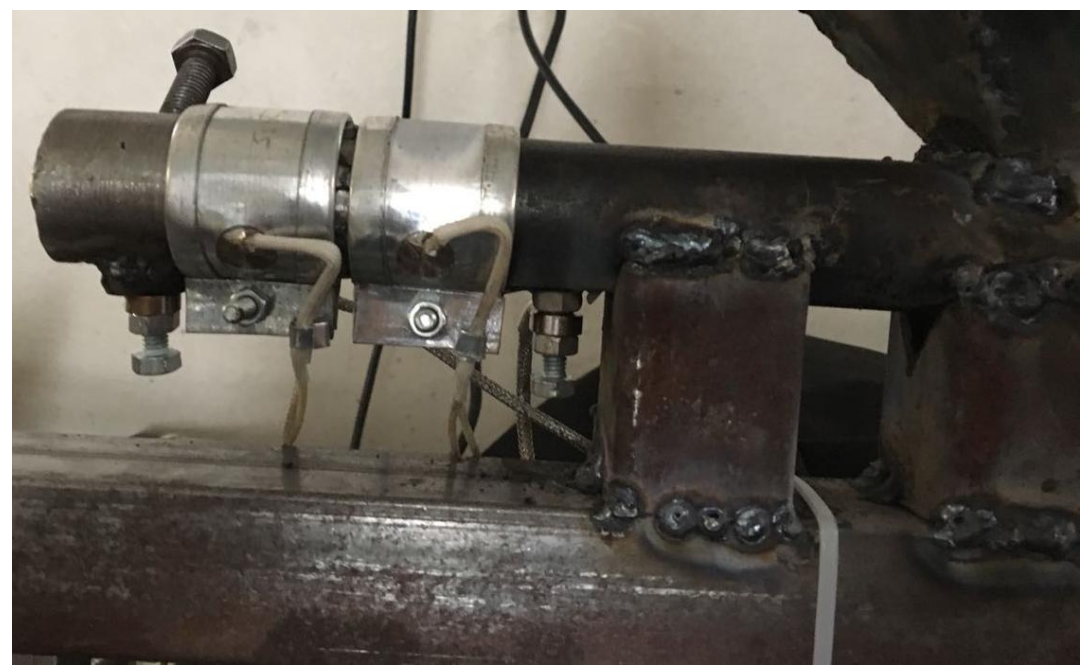

Image 12 Heating Bands

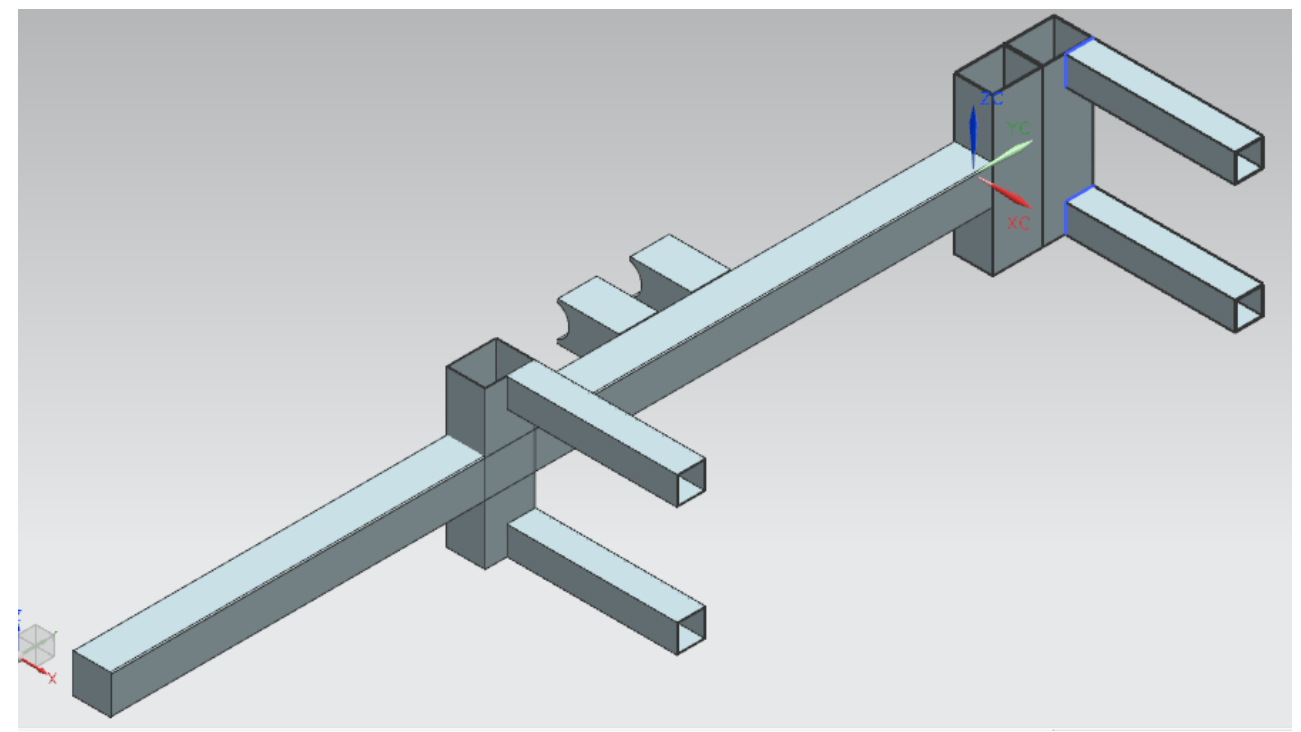

Image 13 Fixing system CAD Model

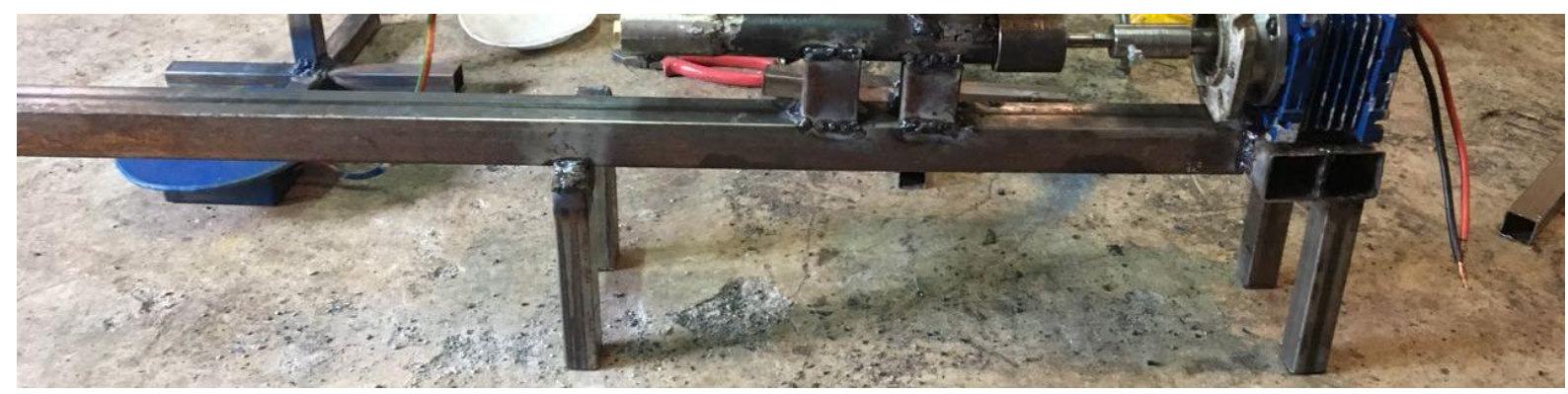

Image 14 Fixing system 


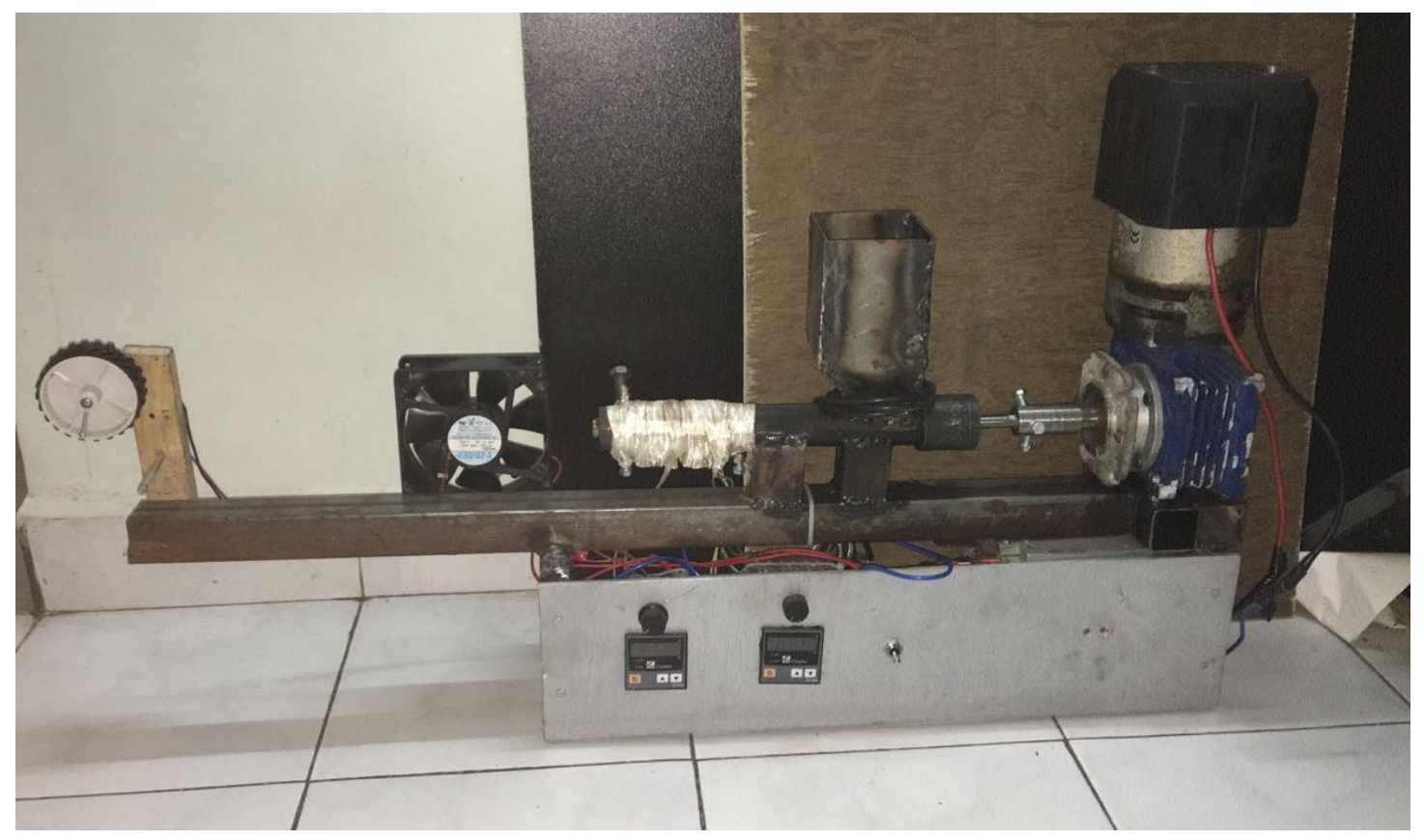

Image 15 ABS Filament Extruder

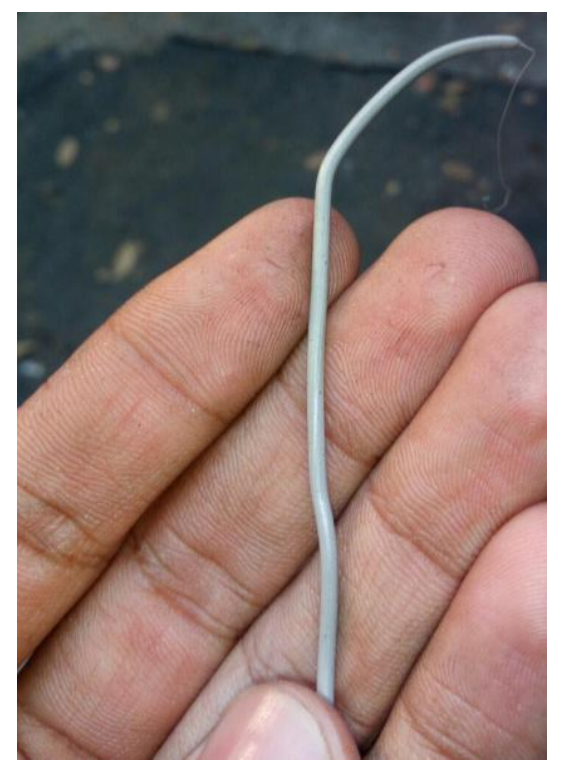

\section{Image 16 ABS Filament Extruder Output}

\section{Conclusion}

The developments in 3D printing have led to the production of objects made from materials such as plastic, metal, paper and even food. This has given end users the opportunity to explore their creativity. 3D printing is being used by universities, manufacturing companies, and everyday users as a quick method of prototyping designs, exploring the capabilities of this technology and seeking ways to improve it. Because of the quick emergence of this technology, leaps have been made towards improving manufacturing.

The development of screw extrusion will hopefully open doors to new ideas for $3 \mathrm{~d}$ printing. Screw extrusion will allow users to have access to a wider variety of materials with a high resolution for their $3 \mathrm{~d}$ printed parts. 


\section{References}

[1] The Definitive Processing Guide and Handbook, by Harold F. Giles, Jr. John R.Wagner, Jr.

[2] Understanding Extrusion, by Chris Rauwendaal. Published by Hanser Publications.

[3] "Parametric analysis and design of straight screw extruder for solids compaction" Journal of King Saud University - Engineering Sciences, March 2017

[4] Single-Screw Extrusion: Introduction and Troubleshooting- Hanser Publication

[5] MASTERING 3D PRINTING, by Johan Horvath. Published by Technology in Action

[6] PRINTING IN PLASTIC, by James Floyd Kelly and Patrick Hood-Daniel. Published by Technology in Action

[7] Dubashi, Jay; Grau, Brian; and McKernan, Alex,"AkaBot 2.0:pet 3Dprinting filament from waste plastic" Mechanical Engineering Senior theses.44, October 2015

[8] Lecture Product Characterization and Processing of Pharmaceutical Particulate Solis, Silo and bunker design for flow, Jurgen Tomas, May 2013

[9] Jimmy Joane, Jorge Gutierrez Edwin Bedoya, "DESIGN FOR PLASIC Extruder", Mechanical Technology School, Pereira - Risaralda, November 2007

[10] http://es.slideshare.net/rbplastics/basic-screw-geometry-things-your-extruder-screwdesigner-never-toldyou-about-screws

[11] http://www.extrudable.me/2013/04/18/exploring-extrusion-variability-and-limits/

[12] https://www.researchgate.net/publication/252894539 